\title{
INTERACTION OF CO2-LASER RADIATION BEAM WITH ELECTRIC ARC PLASMA IN HYBRID (LASER + TIG) WELDING*
}

\author{
I.V. KRIVSTUN ${ }^{1}$, I.V. KRIKENT ${ }^{1}$, V.F. DEMCHENKO ${ }^{1}$, U. REISGEN ${ }^{2}$, A.F. ZABIROV ${ }^{2}$ and O.A. MOKROV ${ }^{2}$ \\ ${ }^{1}$ E.O. Paton Electric Welding Institute, NASU \\ 11 Bozhenko Str., 03680, Kiev, Ukraine. E-mail: office@paton.kiev.ua \\ ${ }^{2}$ Welding and Joining Institute, Aachen University \\ 49 Pontstrasse, Germany. E-mail: reisgen@isf.rwth-aachen.de
}

\begin{abstract}
Self-consistent mathematical model was proposed for the processes of energy-, mass- and charge transfer in the plasma of the column and anode region of electric arc with refractory cathode, running in inert gas, and exposed to a focused beam of $\mathrm{CO}_{2}$-laser radiation, propagating along the arc column. Mathematical description of the processes running in such a system uses single-temperature model of arc plasma, allowing for additional plasma heating due to laser radiation absorption, as well as its multicomponent nature, associated with metal evaporation from cathode surface. Influence of laser beam power and anode surface temperature on thermal and electromagnetic characteristics of plasma of the column and anode region of argon arc on refractory cathode was analyzed. It is shown that as a result of additional local heating of arc plasma by laser radiation, its temperature in the paraxial zone of arc column can rise by 5000-10000 K, compared to the respective arc in TIG welding. This leads to an essential restructuring of spatial distributions of electric potential and density of electric current in the arc column near-anode region, as well as density of thermal flow applied by the arc to the anode, thus changing the distributed and integral characteristics of its thermal and dynamic impact on the metal being welded. It is established that the impact of a focused laser beam on arc plasma leads to the effect of electric current contraction in the center of the region of anode binding of the arc and respective increase of the density of heat flow, applied by the arc to the anode, in hybrid ( $\mathrm{CO}_{2}$-laser + TIG) welding. This promotes an increase of the energy applied to the metal, which is consumed in its melting and, therefore, an increase of penetrability of laser-arc heat source. It is shown that anode metal evaporation leads to a certain weakening of the effect of electric current contraction in the arc near-anode region, which is exposed to a focused laser beam. The influence of laser radiation absorption and refraction in arc plasma on the characteristics of laser beam interacting with it and its thermal impact on the surface of metal being welded under the conditions of hybrid $\left(\mathrm{CO}_{2}\right.$-laser + TIG) welding was studied. It is shown that laser beam absorption in arc plasma leads to a certain decrease of radiation power, reaching the anode surface. The size of beam focusing spot on the above surface practically does not change, i.e. beam refraction in arc plasma turns out to be insignificant. 11 Ref., 1 Table, 14 Figures.
\end{abstract}

Keywords: hybrid ( $\mathrm{CO}_{2}$-laser + TIG) welding, electric arc, refractory cathode, arc column, anode region, evaporating anode, arc plasma, laser beam, simulation

Hybrid laser-arc processes performed by simultaneous action of a focused laser beam and electric arc on the item being welded (within a common heating zone) have become widely accepted over the last decades [1, 2]. At practical implementation of hybrid welding processes a consumableelectrode arc is the most often used (laser + MIG/MAG) [2]. On the other hand, of considerable practical interest are hybrid processes, based on a nonconsumable-electrode arc (laser + TIG), as well as plasma arc (laser + plasma) [1].
Here not only the type of welding arc used in the hybrid process, but also laser beam characteristics, primarily the radiation wave length $\lambda$, are of essential importance. This is associated with a strong dependence of the coefficient of volume absorption of laser radiation in arc plasma $\kappa_{\omega}$ on its wave length. At application of shortwave radiation of solid-body or fiber-optic lasers $(\lambda \approx 1 \mu \mathrm{m})$ in the hybrid process, laser beam absorption in arc plasma is insignificant $\left(\kappa_{\omega} \sim\right.$ $\sim 1 \mathrm{~m}^{-1}$ [1]).

In this case, intensive evaporation of metal from weld pool surface occurs under the impact of high-energy density source of laser heating, which has a significant influence of the compo-

\footnotetext{
* By material of Proceedings of the 7th International Conference «Mathematical Modelling and Information Technologies in Welding and Related Processes» (September 15-9, 2014, Odessa, Ukraine).
} 
sition, thermal, gas-dynamic and electromagnetic properties of arc plasma and, consequently, leads to a change of its thermal and dynamic interaction with the metal being welded. At application of $\mathrm{CO}_{2}$-lasers $(\lambda=10.6 \mu \mathrm{m})$ in hybrid processes both the interaction mechanisms turn out to be significant, namely, laser radiation absorption in arc column plasma $\left(\kappa_{\omega} \sim 100 \mathrm{~m}^{-1}[1]\right)$ and metal evaporation from melt surface under the impact of focused laser beam.

Combining laser and arc heat sources in hybrid $\left(\mathrm{CO}_{2}\right.$-laser + TIG) welding can lead to appearance of synergetic effect, which is manifested in disturbance of additivity of the beam and arc thermal impact on the metal being welded, as well as intensification of the dynamic impact of welding current on the metal pool. As a result, the energy, used for metal melting in hybrid welding, can more than 2 times exceed the sum of the respective energies in welding by each of the heat sources taken separately [1]. Physical factors causing such a synergetic effect are still undisclosed, and should be studied with application of mathematical modeling methods.

Problem definition. The objective of this work is a theoretical study and mathematical modeling of the processes of direct interaction of focused laser radiation with arc plasma. An atmospheric pressure stationary argon arc on refractory tungsten cathode and, in the general case, on evaporating anode from low-carbon steel (iron is the evaporating element) was selected as the object of study, to which a Gaussian beam of continuous $\mathrm{CO}_{2}$-laser radiation focused by an optical system, is applied as shown in Figure 1. As the object, which is the result of such a combination of the laser beam and electric arc, does not have any axial symmetry, we will take the following assumptions to simplify its mathematical model. Let us assume that starting from a certain distance from the anode $\left(z=z_{0}\right)$ the arc column is axially symmetric, and arc plasma characteristics are close to the respective characteristics for an arc on a refractory cathode, located normal to the anode surface. We will also assume that the interaction of the laser beam with the arc plasma proceeds only at $z>z_{0}$. Accordingly, we will introduce a cylindrical system of coordinates $(r, z)$ and will select the calculated areas for arc plasma $\Omega_{\mathrm{p}}=\left\{0 \leq r \leq R_{\mathrm{p}}\right.$, $0 \leq z \leq L\}$ and laser beam $\Omega_{\mathrm{b}}=\left\{0 \leq r \leq R_{\mathrm{b}}, z_{0} \leq\right.$ $\leq z \leq L\}$ (see Figure 1).

Basic equations. We will use the equations of single-temperature model of arc plasma for mathematical description of the processes of energy-, mass- and charge transfer in such a model

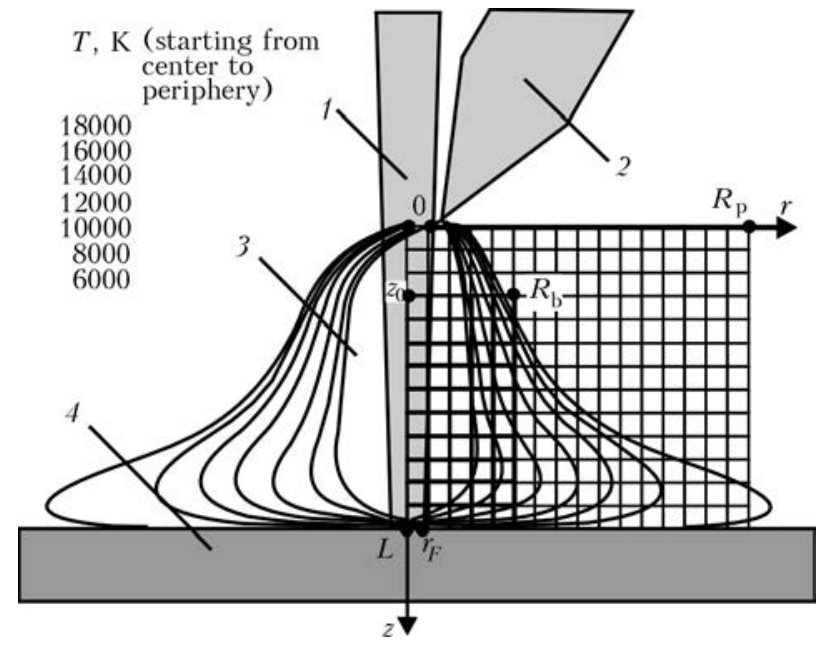

Figure 1. Schematic of combining in the hybrid process a focused laser beam and an electric arc with refractory cathode, as well as temperature isolines for $150 \mathrm{~A}$ argon arc interacting with $1500 \mathrm{~W}$ beam of $\mathrm{CO}_{2}$-laser radiation: 1 laser beam; 2 - refractory cathode; 3 - arc column; 4 metal being welded (anode)

(axially symmetric) system [3, 4]. The respective system of equations should be improved, allowing for the specifics of the studied object and stationarity of the considered processes. Equations of continuity and plasma motion, as well as equations of arc current electromagnetic field are unchanged [3], whereas the equation of energy allowing for additional heating of arc plasma by a focused laser beam should be written as follows [1]:

$$
\begin{gathered}
\rho C_{\mathrm{p}}\left(v \frac{\partial T_{\mathrm{p}}}{\partial r}+u \frac{\partial T_{\mathrm{p}}}{\partial z}\right)=\frac{1}{r} \frac{\partial}{\partial r}\left(r \chi \frac{\partial T_{\mathrm{p}}}{\partial r}\right)+ \\
+\frac{\partial}{\partial z}\left(\chi \frac{\partial T_{\mathrm{p}}}{\partial z}\right)+\frac{k_{\mathrm{B}}}{e}+ \\
+\left\{j_{r} \frac{\partial\left[(5 / 2-\delta) T_{\mathrm{p}}\right.}{\partial r}+j_{z} \frac{\partial\left[(5 / 2-\delta) T_{\mathrm{p}}\right.}{\partial z}\right\}+ \\
+\frac{j_{r}^{2}+j_{z}^{2}}{\sigma}+\kappa_{\omega} S-\psi,
\end{gathered}
$$

where $\rho$ is the plasma mass density; $C_{\mathrm{p}}$ is the specific heat capacity of plasma, allowing for ionization energy; $v, u$ are the radial and axial components of plasma velocity; $T_{\mathrm{p}}$ is the plasma temperature; $\chi$ is the coefficient of plasma temperature conductivity; $k_{\mathrm{B}}$ is the Boltzmann constant; $e$ is the electron charge; $j_{r}, j_{z}$ are the radial and axial components of electric current density in the plasma; $\delta$ is the constant of electron thermodiffusion; $\sigma$ is the specific electric conductivity of plasma; $\psi$ are the energy losses for inherent radiation in the approximation of optically thin plasma. 
In equation (1) energy evolution in arc plasma column due to laser radiation absorption is allowed for by $\kappa_{\omega} S$ term in the right-hand part (where $\kappa_{\omega}$ is the coefficient of volume (back-Bremstrahlung) absorption of laser radiation by arc plasma; $S$ is the spatial distribution of radiation intensity in the laser beam, interacting with plasma).

Let us consider the equation, describing radiation intensity distribution $S(r, z)$ in the laser beam, allowing for its absorption and refraction in non-uniform arc plasma. Assuming that relative change of plasma parameters at distances of the order of laser radiation wave length is small, and that laser radiation reflected from anode surface is absent, quasi-optical approximation was used to describe beam propagation in arc plasma. In this case, the equation for the amplitude of laser beam field assumed to be Gaussian (axially symmetric), can be expressed as follows [1]:

$$
-2 i k \frac{\partial A_{\omega}}{\partial z}=\frac{1}{r} \frac{\partial}{\partial r}\left(r \frac{\partial A_{\omega}}{\partial r}\right)+k^{2}\left(\varepsilon_{\omega}-1\right) A_{\omega},
$$

where $k \equiv k_{z}=2 \pi / \lambda$ is the wave vector of laser radiation; $A_{\omega}^{z}$ is the complex amplitude of laser beam electric field; $\varepsilon_{\omega}=\varepsilon_{\omega}^{\prime}+i \varepsilon_{\omega}^{\prime \prime}$ is the complex dielectric permeability of arc plasma at laser radiation frequency $\omega$.

Sought value $S$ is a time-averaged value of axial component of the density of beam electromagnetic energy flow, and it is related to complex amplitude of electric field $A_{\omega}$ by the relationship [1]

$$
S(r, z)=\frac{1}{2} \sqrt{\frac{\varepsilon^{0}}{\mu^{0}}}\left|A_{\omega}\right|^{2},
$$

where $\varepsilon^{0}$ is the dielectric permeability of vacuum; $\mu^{0}$ is the universal magnetic constant.

To assign the initial distribution of $A_{\omega}\left(r, z_{0}\right)$, let us assume that at $z=z_{0}$ a focused Gaussian laser radiation beam is applied to arc plasma, which in the absence of plasma has minimum radius $r_{F}$ on the anode surface (see Figure 1). Spatial distribution of complex amplitude of an electric field of such a beam at $z \leq z_{0}$ is given by the following expression [1]:

$$
A_{\omega}=A_{\omega F} \frac{r_{F}}{r_{z}} \exp \left[-\frac{r^{2}}{r_{z}^{2}}+i\left(k \frac{r^{2}}{2 R_{z}}-\varphi_{z}\right)\right],
$$

where

$$
\begin{gathered}
r_{z}^{2}=r_{F}^{2}\left[1+\frac{(z-L)^{2}}{z_{F}^{2}}\right] ; \\
R_{z}=(z-L)\left[1+\frac{z_{F}^{2}}{(z-L)^{2}}\right] ; \quad \varphi_{z}=\operatorname{arctg}\left(\frac{z-L}{z_{F}}\right) .
\end{gathered}
$$

Constant $A_{\omega F}$ in (4) is found from integral relationship for the total power of laser radiation in the beam cross-section:

$$
Q(z)=2 \pi \int_{0}^{R_{\mathrm{b}}} S(r, z) r d r,
$$

that allowing for (3) yields

$$
A_{\omega F}=\sqrt{\frac{4 Q^{0}}{\pi r_{F}^{2}}\left(\frac{\mu^{0}}{\varepsilon^{0}}\right)^{1 / 2}},
$$

where $Q_{0}=Q\left(z_{0}\right)$ is the power of initial laser beam.

At numerical simulation of the processes of transfer of energy, pulse, mass and charge in the studied system, let us consider two characteristic variants of the anode, namely, water-cooled (non-evaporating) anode with surface temperature $T_{\mathrm{s}}=500 \mathrm{~K}$ and diffusion-induced evaporating anode. In the latter case we will assume that within the region of the arc anode binding the anode surface temperature $T_{\mathrm{s}}=T_{\mathrm{s}}(r)$ varies by the exponential law [4] and reaches $3000 \mathrm{~K}$ in the center of this region, i.e. does not exceed iron boiling temperature, at which evaporation changes into convective mode. Having thus assigned the anode surface temperature distribution, in order to allow for the multicomponent nature of arc plasma, associated with diffusioninduced evaporation of anode material, the considered model should be complemented by the equation of convective diffusion of ionized metal vapour, describing the transport of neutral atoms, single- and double-charged ions of metal into arc column plasma [4]:

$$
\times c \begin{gathered}
\frac{1}{r} \frac{\partial}{\partial r}\left(r n_{m} v\right)+\frac{\partial}{\partial z}\left(n_{m} u\right)=\frac{1}{r} \frac{\partial}{\partial r} \times \\
\left.r\left[\begin{array}{l}
G_{0} \frac{\partial\left\{n_{m} T_{\mathrm{p}}\right\}}{\partial r}+\bar{G}_{1} \frac{\partial\left\{K_{1} n_{m} T_{\mathrm{p}}\right\}}{\partial r}+ \\
+\bar{G}_{2} \frac{\partial\left\{K_{2} n_{m} T_{\mathrm{p}}\right\}}{\partial r}+\left\{b_{1} K_{1}+b_{2} K_{2}\right\} n_{m} \frac{\partial \varphi}{\partial r}
\end{array}\right]\right)+
\end{gathered}
$$

$$
+\frac{\partial}{\partial z}\left(\left[\begin{array}{l}
G_{0} \frac{\partial\left[n_{m} T_{\mathrm{p}}\right]}{\partial z}+\bar{G}_{1} \frac{\partial\left[K_{1} n_{m} T_{\mathrm{p}}\right]}{\partial z}+ \\
+\bar{G}_{2} \frac{\partial\left[K_{2} n_{m} T_{\mathrm{p}}\right]}{\partial z}+\left\{b_{1} K_{1}+b_{2} K_{2}\right\} n_{m} \frac{\partial \varphi}{\partial z}
\end{array}\right]\right) .
$$

Here, $n_{m}+n_{m 1}+n_{m 2}$ is the total concentration of metal vapour particles in arc plasma; 


$$
\begin{gathered}
\bar{G}_{1}=G_{1}-G_{0} ; \quad \bar{G}_{2}=G_{2}-G_{0} ; \\
K_{1}=\frac{n_{m 1}}{n_{m}} ; \quad K_{2}=\frac{n_{m 2}}{n_{m}} ; \\
G_{Z}=\frac{D_{Z}}{T_{\mathrm{p}}}\left(1-n_{m Z} \frac{M_{m}-\bar{M}_{m Z}}{\rho}\right) ; \quad \bar{M}_{m Z}=\frac{\rho-M_{m} n_{m Z}}{n_{0}-n_{m Z}},
\end{gathered}
$$

$(Z=0,1,2) ; D_{Z}$ are the coefficients of diffusion of atoms $(Z=1)$, of single- $(Z=1)$ and doublecharged $(Z=2)$ metal ions in multicomponent plasma; $M_{m}$ is the metal atom mass; $n_{0}$ is the total concentration of particles in arc column plasma; $b_{Z}=\left(e Z / k_{\mathrm{B}}\right) G_{Z}$ are the mobilities of metal ions $(Z=1,2)$ in the electric field; $\varphi$ is the scalar potential of electric field in arc column.

Equation (8) describes the following kinds of metal vapour particles transfer in arc plasma: convective transfer, concentration diffusion, thermodiffusion, as well as vapour ion drift in the electric field.

Numerical realization of the described model of the processes of transfer of energy, pulse, mass and charge requires assigning the thermodynamic, transport and optical properties of multicomponent arc plasma, depending on its temperature, composition and pressure. For isothermal argon plasma at atmospheric pressure, data given in work [5] were used. Dependencies of thermodynamic and transport properties of $\mathrm{Ar}-$ Fe plasma of different composition were determined by the procedure proposed in [6]. The respective dependencies of energy losses for inherent radiation, coefficient of volume absorption and complex dielectric permeability of arc plasma were calculated as described in [1].

Boundary conditions. We will define the boundary conditions for plasma velocity vector $\vec{V}=\{v, 0, u\}$, its temperature $T_{\mathrm{p}}$, electric field potential $\varphi$, metal vapour particle concentration $n_{m}$ and laser beam field amplitude $A_{\omega}$ as follows. In the initial section of the calculated region we will assign for arc column plasma (plane $z=0$ ) the boundary conditions for values $v, u, T_{\mathrm{p}}, \varphi$ and $n_{m}$ similarly to what was done in $[3,4]$ for an arc with a refractory cathode located normal to the anode surface. In particular, for velocity vector components we will take that

$$
\left.v\right|_{z=0}=0 ;\left.\quad u\right|_{z=0}=u_{0},
$$

where $u_{0}$ value is determined by shielding gas flow rate and diameter of its feed nozzle.

For temperature and electric potential in nearcathode zone of arc column we will assign the following conditions:

$$
\left.T_{\mathrm{p}}\right|_{z=0}=T_{\mathrm{c}}(r) ;\left.\quad \sigma \frac{\partial \varphi}{\partial z}\right|_{z=0}=j_{\mathrm{c}}(r)
$$

where distribution of plasma temperature $T_{\mathrm{p}}(r)$ and electric current density near the cathode $j_{c}(r)$ are selected according to recommendations of [7]. In the zone of shielding gas feed we will take that

$$
\left.T_{\mathrm{p}}\right|_{z=0}=T_{0} ;\left.\quad \frac{\partial \varphi}{\partial z}\right|_{z=0}=0,
$$

where $T_{0}$ is the ambient temperature.

Considering the earlier assumption that laser beam interaction with arc plasma starts at $z=z_{0}$ in equation (1) in region $z<z_{0}$ we will assume that $S=0$, and we will assign the initial condition for equation (2) on the upper boundary of calculated region $\Omega_{\mathrm{b}}$, i.e. at $z=z_{0}$ (see Figure 1 ), as described above.

As regards equation (8), here allowing for the direction of movement of shielding gas and plasma in the near-cathode zone of arc column, we will assume that the particles of evaporated anode metal do not reach plane $z=0$, i.e. on upper boundary of calculated region $\Omega_{\mathrm{b}}$ (see Figure 1) we will take

$$
\left.n_{m}\right|_{z=0}=0 .
$$

For water-cooled (non-evaporating) anode «sticking» conditions are assigned on its surface (plane $z=L$ ), i.e. radial and axial components of plasma flow velocity are taken to be zero. For evaporating anode the near-anode plasma flow, resulting from evaporation of metal atoms from the anode surface and diffusion of ionized metal vapour into the arc column, should be taken into account. Results of calculation performed in [4] show that at $T_{\mathrm{s}}(0)=3000 \mathrm{~K}$, the axial component of the velocity of motion of multicomponent arc plasma near the anode, which is due to diffusioninduced evaporation of its material, can reach the value of the order of $10 \mathrm{~m} / \mathrm{s}$. This requires correction of the boundary condition for normal to anode surface component of plasma velocity vector on anode layer boundary, which in case of diffusion of evaporating anode becomes [4]

$$
\begin{gathered}
\left.u\right|_{z=L}= \\
=\frac{M_{m}\left[Y_{m 0}(r, L)+Y_{m 1_{2}}(r, L)+Y_{m 2_{z}}(r, L)\right]}{\rho(r, L)-M_{m}\left[n_{m 0}(r, L)+n_{m 1}(r, L)+n_{m 2}(r, L)\right.} .
\end{gathered}
$$

Here $Y_{m Z_{z}}(r, L)$ are the radial distributions of axial components of the densities of diffusion flows of metal atoms and ions, being present in the charged state $Z$, on the boundary of the column with anode region of the arc.

On the boundary of column plasma of the arc with the anode layer taken to be infinitely thin 
[8], we can write the following condition of energy balance:

$-\left.\chi \frac{\partial T_{\mathrm{p}}}{\partial z}\right|_{z=L}+j_{\mathrm{a}} \frac{k_{\mathrm{B}}}{e}\left(\frac{5}{2}-\delta\right) T_{\mathrm{pa}}+\Delta \varphi_{\mathrm{a}} j_{\mathrm{a}}+q_{\mathrm{a}}-j_{\mathrm{a}} \bar{\varphi},(14)$

where $j_{\mathrm{a}}=j_{z} \|_{z=L}$ is the absolute value of electric current density on the anode; $\tau_{\mathrm{pa}}=\left.T_{\mathrm{p}}\right|_{z=L}$ is the temperature of arc column on the boundary with the anode region; $\Delta \varphi_{\mathrm{a}}$ is the difference of potential of arc plasma on the outer boundary of the anode layer and potential of anode surface; $q_{\mathrm{a}}$ is the heat flow applied by the arc to the anode; $\bar{\varphi}$ is the work function of anode metal. With good approximation the electric potential of anode surface can be considered to be constant and selected equal to zero, then the boundary condition for plasma potential on the boundary of arc column with the anode layer can be written as [3]

$$
\left.\varphi\right|_{z=L}=\Delta \varphi_{\mathrm{a}} .
$$

To calculate values $\Delta \varphi_{\mathrm{a}}$ and $q_{\mathrm{a}}$ included into boundary conditions (14) and (15), we will use the model of the arc anode region, proposed in [8]. This model allows calculation of radial distributions of heat flow $q_{\mathrm{a}}$, applied by the arc to the anode, as well as a node drop of potential $U_{\mathrm{a}}=-\Delta \varphi_{\mathrm{a}}$ in the region of anode binding of the arc, depending on radial distributions of electric current density on the anode $j_{\mathrm{a}}$ and plasma temperature on anode layer boundary $T_{\mathrm{pa}}$ to be determined.

On the boundary of arc column plasma with anode layer (at $z=L$ ) boundary condition for $n_{m}=n_{m 0}+n_{m 1}+n_{m 2}$ can also be determined according to the model of anode region of the arc with evaporating anode [8], depending on local values of near-anode plasma temperature, anode surface temperature and its evaporation mode.

In the considered diffusion mode of evaporation it can be assumed with sufficient accuracy that local values of partial pressure of atoms and ions of plasma metal component $p_{\mathrm{m}}$ on the mentioned boundary are equal to the pressure of saturated vapour of anode metal at the respective value of its surface temperature $T_{\mathrm{s}}$ :

$$
p_{\mathrm{m}} \mathrm{l}_{z=L}=p_{0} \exp \left\{\frac{\lambda_{\mathrm{v}}}{k_{\mathrm{B}}}\left[\frac{1}{T_{B}}-\frac{1}{T_{\mathrm{s}}(r)}\right]\right\},
$$

where $p_{0}$ is the atmospheric pressure; $\lambda_{\mathrm{v}}$ is the energy spent for transition of one metal atom from the liquid into the vapour phase; $T_{B}$ is the anode metal boiling temperature.

On the system axis of symmetry (at $r=0)$ the boundary conditions for velocity, temperature, plasma electric potential and concentration of metal vapour particles, as well as amplitude of laser beam electric field, assumed to be Gaussian, are assigned in the standard manner (see, for instance, $[1,3,4]$ ):

$$
\begin{gathered}
\left.v\right|_{r=0}=0 ;\left.\quad \frac{\partial u}{\partial r}\right|_{r=0}=0 ;\left.\quad \frac{\partial T_{\mathrm{p}}}{\partial r}\right|_{r=0}=0 ; \\
\left.\frac{\partial \varphi}{\partial r}\right|_{r=0}=0 ;\left.\quad \frac{\partial n_{m}}{\partial r}\right|_{r=0}=0 ;\left.\quad \frac{\partial A_{\omega}}{\partial r}\right|_{r=0}=0 .
\end{gathered}
$$

On the outer boundary of calculated region for plasma (at $r=R_{\mathrm{p}}$ ) we will express boundary conditions for velocity and electric potential as follows:

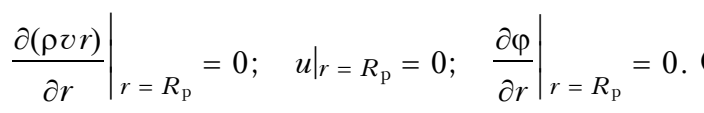

We will determine boundary conditions for temperature and concentration of metal vapour particles at $r=R_{\mathrm{p}}$, depending on the direction of plasma flow motion [3, 4]:

$$
\begin{aligned}
& \left.T_{\mathrm{p}}\right|_{r=R_{\mathrm{p}}}=T_{0} ;\left.\quad n_{m}\right|_{r=R_{\mathrm{p}}}=0 \text { at }\left.\vartheta\right|_{r=R_{\mathrm{p}}} \leq 0 ; \\
& \left.\frac{\partial T_{\mathrm{p}}}{\partial r}\right|_{r=R_{\mathrm{p}}}=\left.\frac{\partial n_{m}}{\partial r}\right|_{r=R_{\mathrm{p}}}=0 \text { at }\left.v\right|_{r=R_{\mathrm{p}}}>0 .
\end{aligned}
$$

Finally, assuming that the transverse dimension of the laser beam is essentially smaller than the radius of the respective calculated region $R_{\mathrm{b}}$, we will write

$$
\left.A_{\omega}\right|_{r=R_{\mathrm{b}}}=0 .
$$

System of diffusion equations, which describe the transfer of energy, pulse, mass and charge in multicomponent arc plasma, exposed to a focused laser beam, with the above-mentioned boundary conditions, was solved numerically, using a combined Lagrangian-Eulerian method [9, 10], adapted to the conditions of a compressible medium. Parabolic equation (2) for complex amplitude of laser beam field was solved by the method, described in detail in [1].

Modeling results. Numerical modeling of the characteristics of plasma column and anode region of electric arc with tungsten cathode and steel anode, exposed to a focused beam of $\mathrm{CO}_{2}{ }^{-}$ laser radiation, was conducted at the following system parameters: length of interelectrode gap $L=4 \mathrm{~mm}$; arc current $I=150 \mathrm{~A}$; shielding gas was argon, pressure was atmospheric; initial laser beam power $Q^{0}$ was varied in the range of 500$1500 \mathrm{~W}$; beam radius in the focusing plane $(z=$ $=L) r_{F}=0.2 \mathrm{~mm}$; distance from cathode to the 


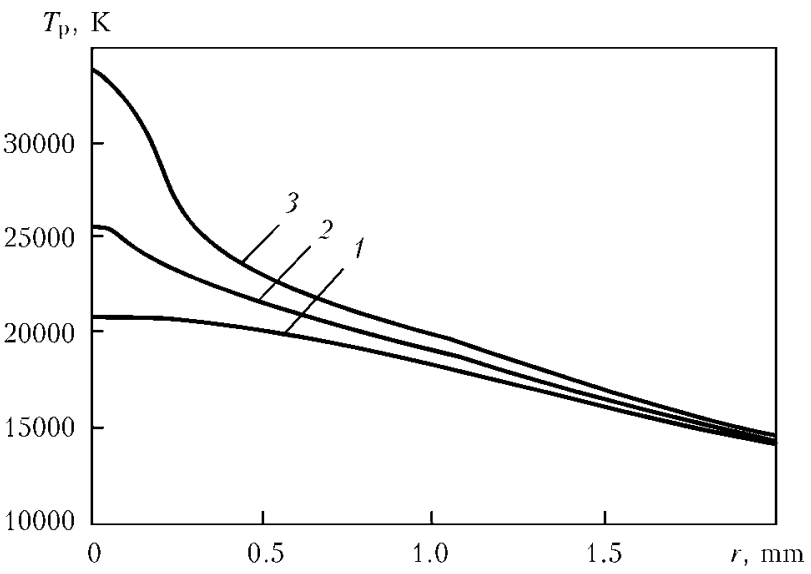

Figure 2. Radial distributions of plasma temperature in the cross-section $z=2.5 \mathrm{~mm}$ of arc column at $T_{\mathrm{S}}=500 \mathrm{~K}: 1-$ $Q^{0}=0 ; 2-500 ; 3-1500 \mathrm{~W}$

plane, where the interaction of the laser beam with arc plasma begins, $z_{0}=1 \mathrm{~mm}$ was selected.

Arc plasma. Let us first consider the influence of laser radiation absorption by arc plasma on the thermal and electric characteristics of the column and anode region of the studied arc. Under the impact of the focused beam of $\mathrm{CO}_{2}$-laser radiation a high-temperature region localized near the beam axis forms in the arc column ( $\mathrm{Fi}^{-}$ gure 2), in which the maximum plasma temperature increases with the increase of laser beam power. Here, the radius of the above-mentioned region is equal to a value of the order of $0.5 \mathrm{~mm}$. So, on the axis of $150 \mathrm{~A}$ arc (at $z=2.5 \mathrm{~mm}$ ) plasma temperature in the absence of laser impact, is equal to $20,700 \mathrm{~K}$, whereas under the impact of laser beam of power $Q^{0}=500$ and $1500 \mathrm{~W}$ it increases up to 25,100 and $32,800 \mathrm{~K}$, respectively.

The effect of local laser heating of arc plasma is manifested right up to the boundary of the column with the arc anode region, leading to a noticeable increase of axial values of near-anode plasma temperature. Influence of arc plasma heating by the laser beam on its temperature distribution along the axis of arc column (laser beam axis) is shown in Figure 3. Note that the anode surface temperature (its material evaporation) practically does not influence the spatial distribution of plasma temperature in the arc column that is in keeping with the conclusions of [4].

Change of spatial distribution of arc plasma temperature under the impact of focused laser beam radiation causes a noticeable redistribution of electric current density in the discharge. Figure 4 gives the distributions of absolute value of axial component of current density along the arc axis, exposed to the impact of $\mathrm{CO}_{2}$-laser radiation beam of different power (curve $1-Q^{0}=$ $=1500 \mathrm{~W} ; 2-500 \mathrm{~W}$ ), compared to the respec-
$T_{\mathrm{p}}, \mathrm{K}$

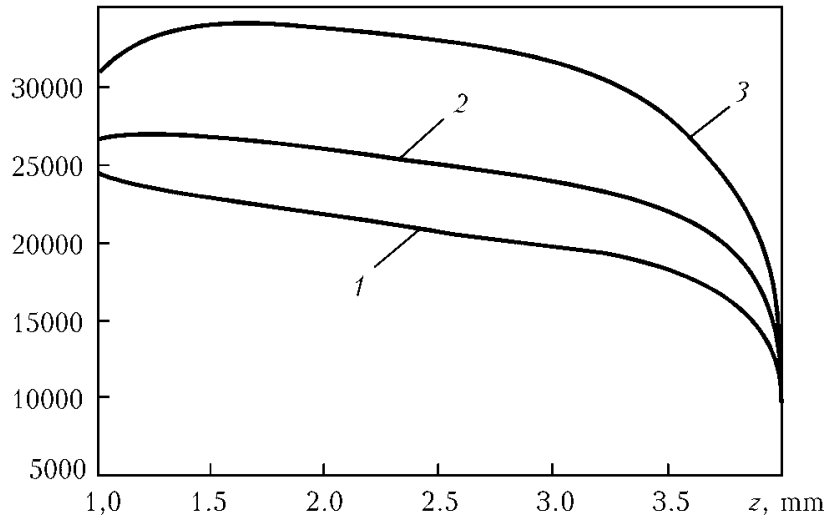

Figure 3. Axial distributions of plasma temperature on the axis of arc column at $T_{\mathrm{S}}=500 \mathrm{~K}: 1-Q^{0}=0 ; 2-500$; $3-1500 \mathrm{~W}$

tive distribution $\left|j_{z}(0, z)\right|$ for the regular arc (curve 3).

Despite the fact that plasma temperature in the axial zone of the arc column rises significantly under the impact of laser radiation (Figures 2 and 3), electric current density on the discharge axis in the initial sections of the region of laserarc interaction $(0<z<3 \mathrm{~mm})$ remains practically the same as for the regular arc. Influence of laser heating of arc plasma on current density distribution in the arc column starts to be manifested closer to the anode. In the near-anode zone of the column value $j_{z}(0, z) \mid$ for an arc exposed to laser radiation, increases significantly, the maximum value of current density on the arc axis rising with increase of initial beam power (see Figure 4). As a result, for instance, at the impact of $1500 \mathrm{~W}$ laser beam on $150 \mathrm{~A}$ arc, current density in the center of the region of anode binding of the arc can more than 3 times exceed the respective value for the regular arc at other conditions being equal (Figure 5).

Heat flow $q_{\mathrm{a}}$ applied by the arc to the anode, essentially depends on the density of electric cur-

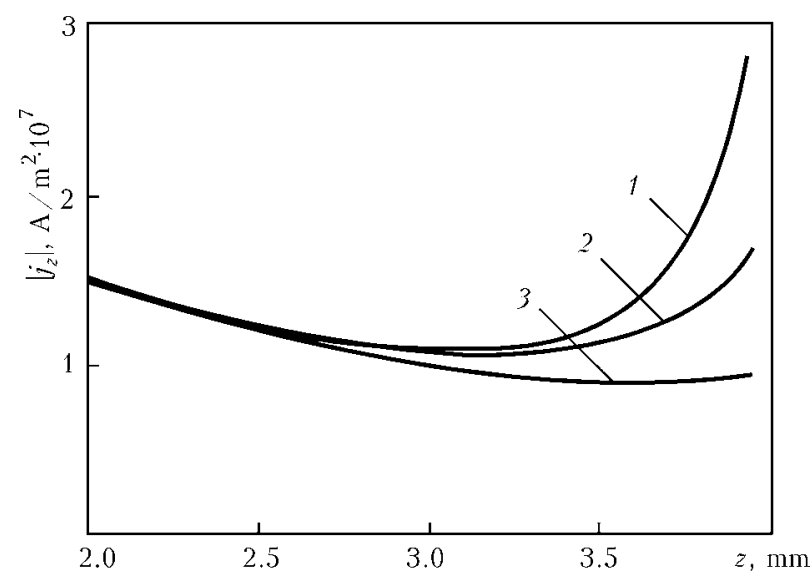

Figure 4. Distributions of absolute value of axial component of electric current density on the arc axis along its column at $T_{\mathrm{S}}=500 \mathrm{~K}$ (for $1-3$ see the text) 


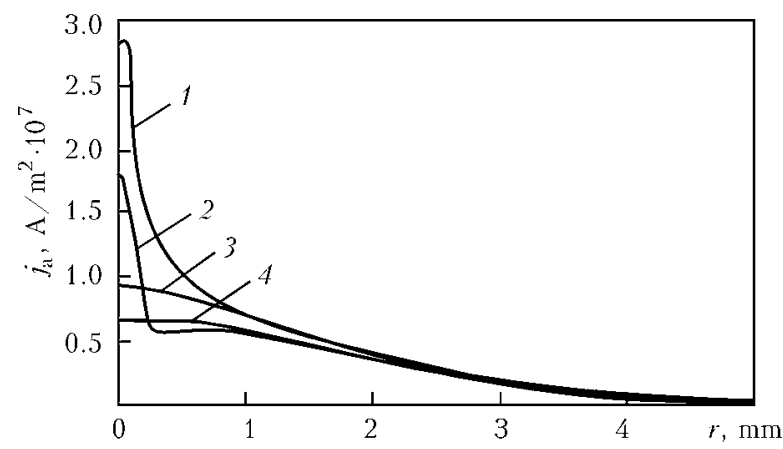

Figure 5. Distributions of electric current density on the anode: $1-Q^{0}=1500 \mathrm{~W}, T_{\mathrm{s}}=500 \mathrm{~K} ; 2-Q^{0}=1500 \mathrm{~W}$, $T_{\mathrm{S}}(0)=3000 \mathrm{~K} ; 3-Q^{0}=0, T_{\mathrm{S}}=500 \mathrm{~K} ; 4-Q^{0}=0$, $T_{\mathrm{S}}(0)=3000 \mathrm{~K}$

rent on the anode [3, 4], therefore, the nature of $q_{\mathrm{a}}(r)$ distribution, given in Figure 6, is similar to distribution of current density on the anode (see Figure 5). So, at the impact of $1500 \mathrm{~W}$ laser beam on $150 \mathrm{~A}$ arc, axial value of density of the heat flow, applied by the arc to the anode, increases almost 4 times. At increase of anode temperature (allowing for its evaporation) the effect of contraction of electric current and density of heat flow on the anode, respectively, becomes somewhat weaker, remaining, however, significant compared to an arc, not exposed to laser radiation (see Figures 5 and 6).

To analyze the effect of arc contraction near the anode surface, we will consider radial distributions of near-anode plasma temperature $T_{\mathrm{pa}}$ (Figure 7) and drops of electric field potential $\Delta \varphi_{\mathrm{a}}$ on the anode layer (Figure 8) for an arc exposed to a laser beam of power $Q^{0}=1500 \mathrm{~W}$ (curves 1) and for a regular arc (curves 2 in the Figure).

It is known [8] that anode voltage $\operatorname{drop} U_{\mathrm{a}}=$ $=-\Delta \varphi_{\mathrm{a}}$ is negative, dependent on density of current on the anode, anode and near-anode plasma temperatures, and in such a way that potential jump $\Delta \varphi_{\mathrm{a}}$ rises noticeably with increase of plasma temperature near the anode and weakly decreases at increase of current density on the anode. At $Q^{0}=1500 \mathrm{~W}$ the axial temperature of plasma on

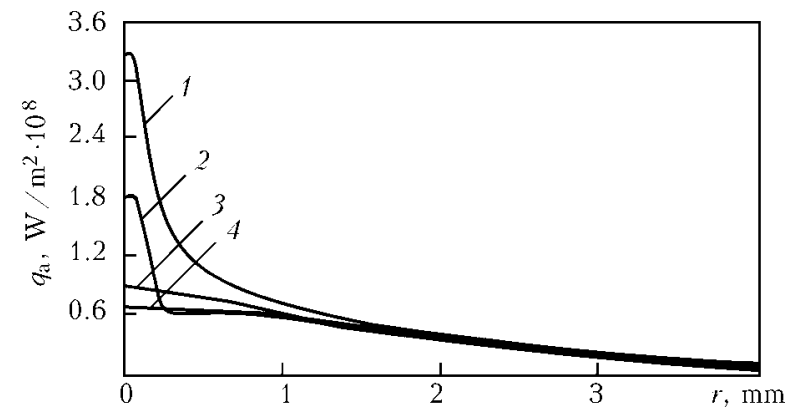

Figure 6. Distributions of heat flow contributed by arc to the anode over its surface: $1-Q^{0}=1500 \mathrm{~W}, T_{\mathrm{s}}=500 \mathrm{~K}$; $2-Q^{0}=1500 \mathrm{~W}, T_{\mathrm{S}}(0)=3000 \mathrm{~K} ; 3-Q^{0}=0, T_{\mathrm{S}}=$ $=500 \mathrm{~K} ; 4-Q^{0}=0, T_{\mathrm{s}}(0)=3000 \mathrm{~K}$

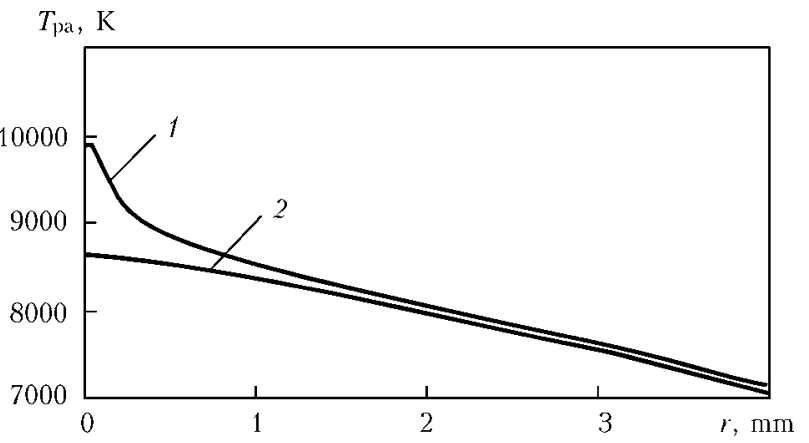

Figure 7. Radial distributions of plasma temperature on the anode layer boundary at $T_{\mathrm{S}}=500 \mathrm{~K}$ (for 1,2 see the text)

the anode layer boundary for an arc with a nonevaporating anode rises by almost $1200 \mathrm{~K}$, compared to a regular arc (see Figure 7). It results in increase of axial values of plasma potential on the above boundary and appearance of a pronounced maximum near the arc axis in $\Delta \varphi_{\mathrm{a}}(r)$ distribution (see Figure 8, curve 1). An abrupt change of $\Delta \varphi_{\mathrm{a}}$ along the anode surface leads to deformation of equal potential lines in paraxial zone of near-anode plasma, resulting in appearance of a significant radial component of electric field intensity vector and respective radial component of current density. Alongside increase of temperature (plasma electric conductivity), as well as axial component of electric field intensity in the above zone, it leads to an essential contraction of electric current in the region of anode binding of the arc exposed to a laser beam (see Figure 5), compared to a regular arc.

The above features of the influence of radial distribution of anode drop of potential on electric current distribution in the plasma near the anode, are illustrated in Figures 9 and 10, which give the equipotential lines and vector fields of current density in near-anode plasma for a regular arc and for an arc, exposed to focused laser radiation. Note that in the case of an anode evaporating under the impact of diffusion $\left(T_{\mathrm{s}}(0)=\right.$ $=3000 \mathrm{~K})$ plasma temperature on the boundary with the arc anode region decreases compared to water-cooled (non-evaporating) anode both in

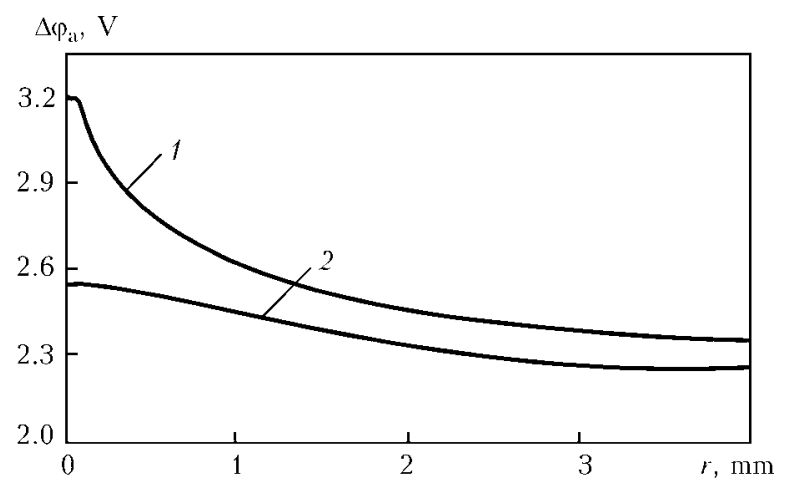

Figure 8. Radial distributions of electric potential drop in the arc anode layer at $T_{\mathrm{s}}=500 \mathrm{~K}$ (for 1,2 see the text) 


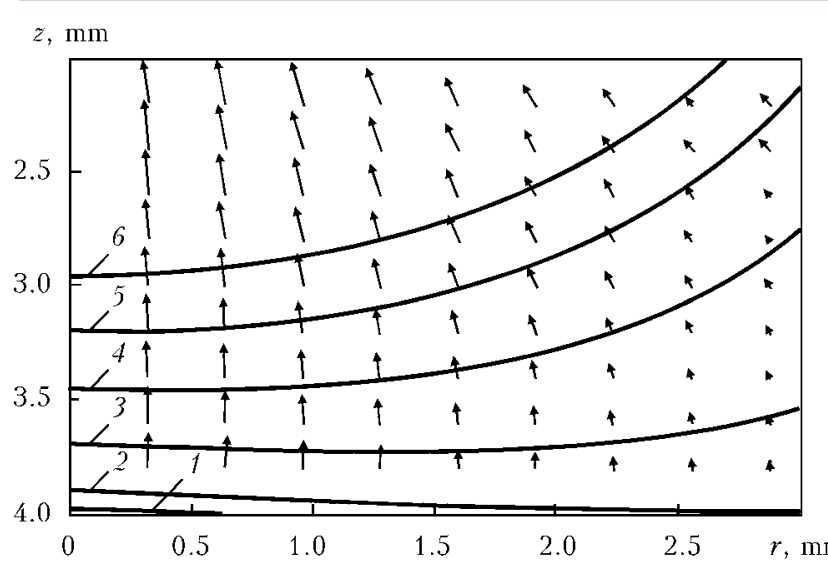

Figure 9. Equal potential lines and vector field of electric current densities in near-anode plasma of regular arc at $T_{\mathrm{S}}=$ $500 \mathrm{~K}: 1-\varphi=2.5 ; 2-2.25 ; 3-2 ; 4-1.75 ; 5-$ $1.5 ; 6-1.25 \mathrm{~V}$

the presence, and in the absence of laser impact. As a result, the effect of arc current contraction under the laser beam impact becomes less noticeable (see Figure 5).

Laser beam. Let us consider the influence of arc column plasma on the laser beam interacting with it. Let $\zeta(z)=Q(z) / Q^{0}$ be the coefficient of beam weakening due to its absorption in arc plasma, where $Q(z)$ is the laser beam power in the section of the arc with axial coordinate $z$, calculated by formula (6). Because of beam absorption in arc column, about $74 \%$ of laser beam power reaches the anode surface (Figure 11). This value only slightly depends on initial beam power and anode surface temperature for the selected parameters of the laser beam and the arc.

The power applied by laser beam to the anode $Q_{\mathrm{b}}=\Gamma Q(L)$ can be assessed, knowing the power of radiation $Q(L)$, which has reached the anode, and the coefficient of laser radiation absorption by its surface $\Gamma$. Assuming that the anode is watercooled, made from low-carbon steel, and select-

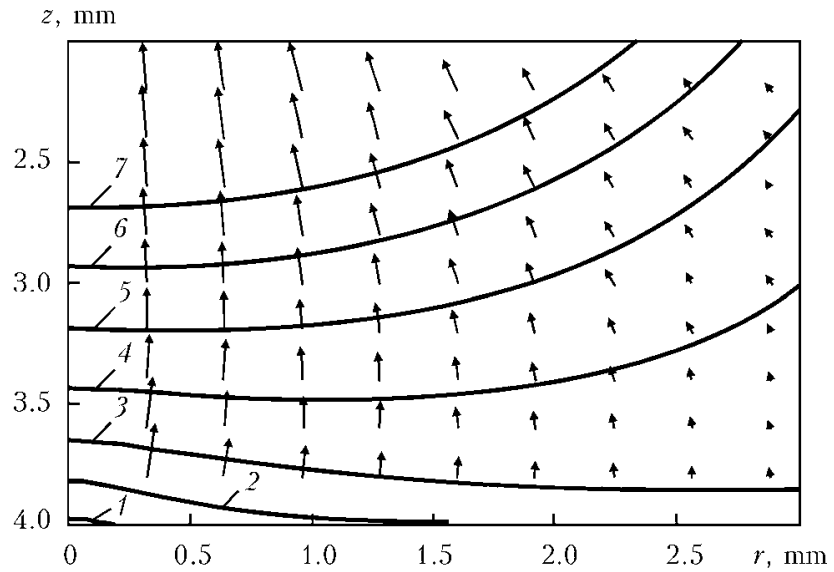

Figure 10. Equal potential lines and vector field of electric current densities in near-anode plasma of regular arc $\left(T_{\mathrm{S}}=\right.$ $=500 \mathrm{~K})$, exposed to laser beam of $1.5 \mathrm{~kW}$ power: 1 $\varphi=3 ; 2-2.5 ; 3-2.25 ; 4-2 ; 5-1.75 ; 6-1.5$; $7-1.25 \mathrm{~V}$

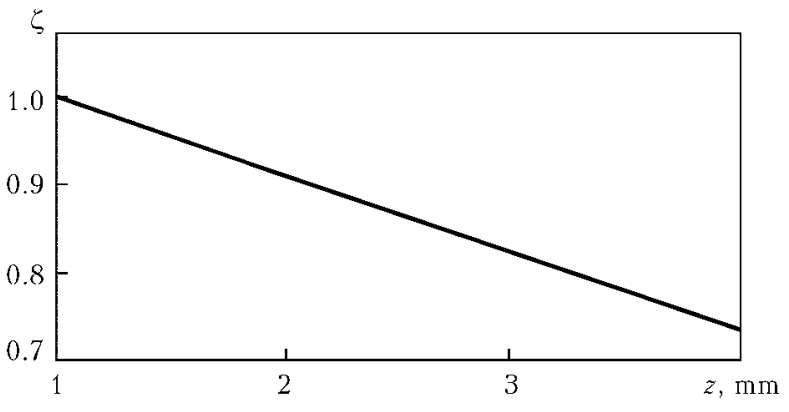

Figure 11. Change of coefficient of laser radiation weakening in arc plasma along the arc length

ing $\Gamma=0.1$ [11], we obtain $Q_{\mathrm{b}}=110 \mathrm{~W}$ at $Q^{0}=$ $=1500 \mathrm{~W}$. Note that melting and intensive laser-induced evaporation of the anode metal can lead to formation of a vapour-gas channel, which can absorb practically all the laser radiation power reaching the anode surface. Here the power, applied to the anode by the laser beam at $Q^{0}=1500 \mathrm{~W}$, can reach value $Q_{\mathrm{b}}=1105 \mathrm{~W}$ and can be commensurate with the power imparted to such an anode by the arc.

Distribution of radiation intensity along the axis of focused laser beam, interacting with arc plasma, compared to the respective distribution for the initial beam, is shown in Figure 12. Unlike laser beam propagating in air (in the absence of the arc), at propagation in arc plasma the intensity of laser radiation on beam axis $S_{0}=S(0, z)$ decreases when coming closer to the anode surface.

As follows from calculated dependencies, given in Figures 11 and 12, interaction of the focused laser beam with arc plasma under the considered conditions is mainly reduced to absorption of beam energy by the plasma, here the role of its refraction in non-uniform arc plasma turns out to be insignificant. This is also indicated by the dependence of effective beam radius $r_{\mathrm{b}}(z)$ (Figure 13), determined as distance from its axis, at which radiation intensity is equal to $1 \%$ of the respective $S(0, z)$ value. Effective radius of the laser beam, interacting with plasma, practically does not differ from initial beam radius in

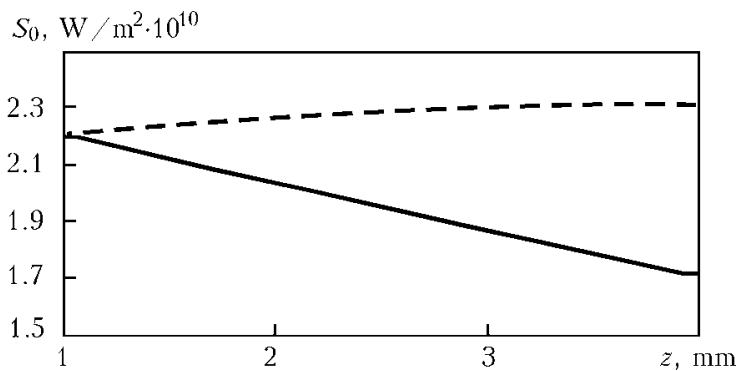

Figure 12. Distribution of radiation intensity on the axis of focused laser beam of $1500 \mathrm{~W}$ power propagating in arc plasma (solid curve), and for initial beam (hatched) 


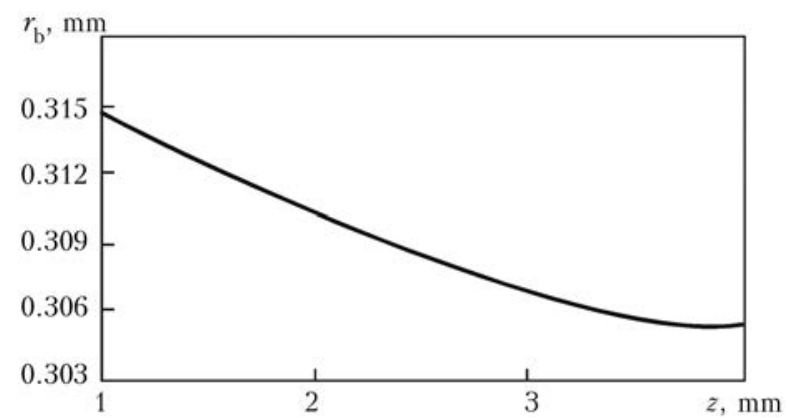

Figure 13. Distribution of laser beam effective radius along its length

the respective section, and for conditions considered in this work, it does not depend on its power.

Discussion. The predicted by mathematical modeling effect of contraction of electric current on the anode and heat flow to the anode, respectively, arising under the impact of focused beam of $\mathrm{CO}_{2}$-laser radiation on argon arc with refractory cathode, can have important technological consequences. Under the impact of $1500 \mathrm{~W}$ laser beam on $150 \mathrm{~A}$ arc, axial values of the above characteristics increase by more than 3 times. Thus, laser impact on the considered arc makes it a higher energy density source of thermal and dynamic impact on the metal. Calculations show that additional laser heating of arc plasma also causes a marked increase of total power applied by the arc to the anode $Q_{\mathrm{a}}=2 \pi \int_{0}^{R_{\mathrm{p}}} q_{\mathrm{a}} r d r$ (the

Table), and with increase of laser beam power the total heat flow contributed by the arc to the anode increases both for the water-cooled anode and for the anode evaporating through diffusion.

Now let us consider the mechanism of synergetic effect arising at combining of laser and arc heat sources under the conditions of hybrid $\left(\mathrm{CO}_{2}\right.$ laser + TIG) welding. This effect is manifested in disturbance of additivity of thermal impact of the laser beam and arc plasma on the metal being welded, which may result in the energy used for metal melting in hybrid welding, being more than 2 times greater than the sum of the respective

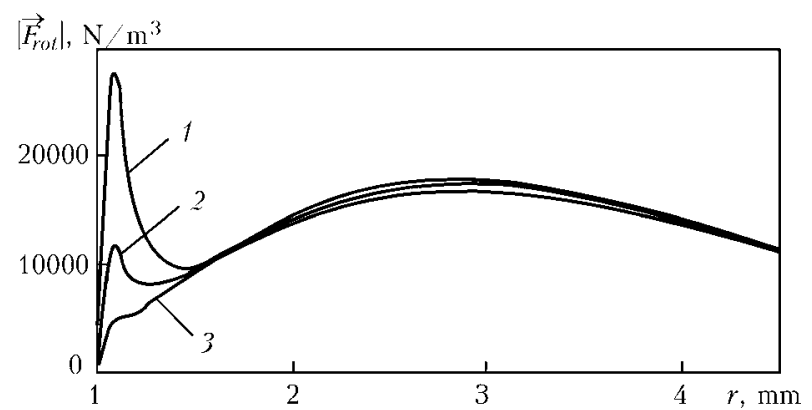

Figure 14. Distribution of eddy component of Lorentz force over the anode surface at $T_{\mathrm{S}}=500 \mathrm{~K}: 1-Q^{0}=1500 ; 2-$ $500 \mathrm{~W} ; 3$ - regular arc
Total heat flow into the anode from the arc depending on laser radiation power and thermal state of anode surface

\begin{tabular}{|c|c|c|}
\hline$T_{\mathrm{s}}(0), \mathrm{K}$ & $Q^{0}, \mathrm{~W}$ & $Q_{\mathrm{a}}, \mathrm{W}$ \\
\hline 500 & 0 & 1431.8 \\
\hline 500 & 500 & 1494.0 \\
\hline 500 & 1500 & 1564.4 \\
\hline 3000 & 0 & 1478.6 \\
\hline 3000 & 500 & 1526.1 \\
\hline 3000 & 1500 & 1579.7 \\
\hline
\end{tabular}

energies in welding by each individually taken heat source [1].

It is known that in an axially symmetric magnetic field of arc current, molten metal motion under the impact of the Lorentz force is excited by its eddy component $\vec{F}_{\text {rot }}$ having centripetal direction. Magnitude of this force is maximum on weld pool surface and decreases at electric current spreading in the volume of metal being welded. The thus arising pressure gradient causes a descending melt flow transporting the overheated metal from the pool surface to the melting front. At intensive flow of the melt towards the weld pool bottom part, the effectiveness of consumption (use for metal melting) of thermal power, applied to the anode by both the heat sources is increased, and the molten metal volume becomes greater, respectively. Let us approximately evaluate the influence of laser-arc interaction on distribution of eddy component of electromagnetic force on the surface of anode molten metal, assuming it to be undeformable. Arc contraction, which is expressed in an essential increase of electric current density on the metal surface near the center of the region of arc anode binding (see Figure 5), leads to an abrupt increase of the centripetal component of the Lorentz force in the above-mentioned zone ( $\mathrm{Fi}^{-}$ gure 14). The dynamic effect due to contraction of arc current on the anode surface, turns out to be the higher, the greater the laser radiation power.

The conducted approximate evaluation of electromagnetic force applied to the weld pool metal, requires further precising, primarily aimed at studying the thermal, electromagnetic and gasdynamic processes at arcing on the surface of molten anode metal, deformed under the impact of a high energy density laser source.

\section{Conclusions}

1. Additional local heating of arc plasma by a focused laser beam results in formation in the arc column of a high-temperature region localized 
near the beam axis, in which the maximum plasma temperature rises with the increase of laser radiation power. It is anticipated that formation of such a rigidly coupled with the laser beam axis, high-temperature region of arc plasma, having a high conductivity, will increase the spatial stability of the arc under the conditions of the hybrid process $\left(\mathrm{CO}_{2}\right.$-laser + TIG) that is particularly important at large welding speeds.

2. Redistribution of electric potential in arc plasma, occurring due to variation of spatial distribution of its temperature under the impact of the focused laser beam, leads to an essential increase of current density in near-anode plasma, as well as density of heat flow, applied by the arc to the anode, making such an arc a higher energy density source of thermal and electromagnetic impact on the metal being welded. Total power, applied by the arc to the metal in hybrid $\left(\mathrm{CO}_{2}\right.$-laser + TIG) welding, exceeds the power contributed by the respective arc under the conditions of regular nonconsumable-electrode welding. The effect predicted by the computational experiment of arc current contraction in hybrid welding may lead to a significant increase of the Lorentz force, transporting overheated metal from the center of the region of arc anode binding on the weld pool surface to its bottom, that should lead to increase of the power fraction of each of the used heat sources, which is consumed for melting of the metal being welded.

3. Interaction of the focused beam of $\mathrm{CO}_{2}$-laser radiation with plasma of the argon arc with refractory cathode leads to a certain decrease of the power of radiation, reaching the anode surface, as a result of partial absorption of laser radiation in the arc column plasma. Here, refraction of the beam in nonuniform arc plasma turns out to be negligible. The power applied by the laser beam to the metal, being welded in hybrid $\left(\mathrm{CO}_{2}\right.$-laser + TIG $)$ welding, can be both greater and smaller than the power applied to the metal in laser welding, depending on the conditions of absorption of laser radiation reaching the metal surface, determined, for instance, by the possibility of formation of a vapour-gas channel in the weld pool, which more effectively absorbs laser radiation than the flat surface of the melt.

This work was performed under Joint Project RE-2755/20-1 «Self-consistent modeling of hybrid (laser-TIG) and (laser-plasma) welding in the heat-conductivity penetration mode» funded by the German Research Society (DFG). The authors express their gratitude to DFG for financial support of these studies.

1. Seyffarth, P., Krivtsun, I.V. (2002) Laser-arc processes and their applications in welding and material treatment. Vol. 1. London: Taylor and Francis Books.

2. Dilthey, U. (2002) Laser arc hybrid welding - An overview. Arc welding processes and production systems: IIW Doc. XII-1710, 243-248.

3. Krikent, I.V., Krivtsun, I.V., Demchenko, V.F. (2012) Modelling of processes of heat-, mass- and electric transfer in column and anode region of arc with refractory cathode. The Paton Welding J., 3, $2-6$.

4. Krikent, I.V., Krivtsun, I.V., Demchenko, V.F. (2014) Simulation of electric arc with refractory cathode and evaporating anode. Ibid., 9, 17-24.

5. Boulos, M.I., Fauchais, P., Pfender, E. (1997) Thermal plasmas: Fundamentals and applications. Vol. 1. New York, London: Plenum Press.

6. Krivtsun, I.V., Porytsky, P., Demchenko, V. et al. (2010) On the application of the theory of Lorentzian plasma to calculation of transport properties of multicomponent arc plasmas. Europ. Phys. J. $D, \mathbf{5 7}, 77-85$.

7. Wendelstorf, J., Simon, G., Decker, I. et al. (1997) Investigation of cathode spot behaviour of atmospheric argon arcs by mathematical modeling. In: Proc. of 12th Int. Conf. on Gas Discharges and Their Applications (Germany, Greifswald, 1997), Vol. 1, 62-65.

8. Krivtsun, I.V., Demchenko, V.F., Krikent, I.V. (2010) Model of the processes of heat, mass and charge transfer in the anode region and column of the welding arc with refractory cathode. The Paton Welding J., 6, 2-9.

9. Lyashko, I.I., Demchenko, V.F., Vakulenko, S.A. (1981) Version of the method of splitting equations of uncompressible viscous fluid dynamics on Lagrangian-Eulerian networks. Doklady AN UkrSSR, Series A, 7, 43-47.

10. Demchenko, V.F., Lesnoj, A.B. (2000) LagrangianEulerian method of numerical solution of multidimensional problems of convective diffusion. Dopovidi NANU, 11, 71-75.

11. Gladush, G.G., Smurov, I. (2011) Physics of laser materials processing: Theory and experiment. Berlin, Heidelberg: Springer.

Received 19.01.2015 